\title{
Distortion Risk Measures for Sums of Random Variables
}

\author{
Grzegorz Darkiewicz, Jan Dhaene, and Marc Goovaerts (Leuven)
}

\section{Introduction}

Distortion risk measures were introduced in Wang (1996). For a given non-decreasing function $g:[0,1] \rightarrow[0,1]$ such that $g(0)=0$ and $g(1)=1$ for every risk the corresponding risk measure is defined as follows:

$$
H_{g}[X]=\int_{0}^{\infty} g\left(1-F_{X}(t)\right) d t=\int_{0}^{1} F_{X}^{-1}(1-q) d g(q),
$$

where $F_{X}(t)$ denotes the distribution function of $X \geq 0$.

The distortion risk measures have some useful properties, e.g. positive homogeneity, translation invariance, additivity for comonotonic risks, preservation of stochastic dominance. Moreover if one additionally assumes concavity of the distortion function $g$ than the corresponding risk measure will be also subadditive.

These properties of distortion risk measures have been comprehensively studied in many works (see e.g. Wang (1996), Wang et. al (1998), Wang and Young (1998), Wirch and Hardy (2000), Dhaene et. al (2004)). In this contribution we investigate the behavior of distortion risk measures when applied to sums of random variables against some well-known dependency measures between summands (we assume that the marginal distributions are fixed). The theorem we cite below states that when the dependency level differs strongly (which is expressed in the terms of the so-called correlation order of pairs of random variables) and the distortion function is concave (which implies the aversion towards risk well-known from the utility theory), then the corresponding risk measure should behave consistently with increasing dependence between the variates in the sum, and increase.

Definition 1 Let $\left(X_{1}, Y_{1}\right)$ and $\left(X_{2}, Y_{2}\right)$ be elements of $R\left(F_{X}, F_{Y}\right)$ (i.e. have the same marginal distributions equal to $F_{X}$ and $\left.F_{Y}\right)$. Then we say that $\left(X_{1}, Y_{1}\right)$ precedes $\left(X_{2}, Y_{2}\right)$ in correlation order when either of the two equivalent conditions holds:

(a) for all non-decreasing functions $f, g$ one has that $\operatorname{Cov}\left(f\left(X_{1}\right), g\left(Y_{1}\right)\right) \leq$ $\operatorname{Cov}\left(f\left(X_{2}\right), g\left(Y_{2}\right)\right)$, provided that the respective covariance functions exist.

(b) for all pairs $(x, y) F_{\left(X_{1}, Y_{1}\right)}(x, y) \leq F_{\left(X_{2}, Y_{2}\right)}(x, y)$.

We denote the correlation order by $\leq_{c o r r}$, which is equivalent to ordering of copulas.

Theorem 1 Suppose that $g$ is a non-decreasing concave function such that $g(0)=0$ and $g(1)=1$. Assume $\left(X_{1}, Y_{1}\right),\left(X_{2}, Y_{2}\right) \in R\left(F_{X}, F_{Y}\right)$ are such that $\left(X_{1}, Y_{1}\right) \leq_{\text {corr }}$ $\left(X_{2}, Y_{2}\right)$. Let $S_{1}=X_{1}+Y_{1}$ and $S_{2}=X_{2}+Y_{2}$. Then $H_{g}\left[S_{1}\right] \leq H_{g}\left[S_{2}\right]$. 
Proof See Wang \& Dhaene (1998).

However the correlation order defines only a partial order in the class $R\left(F_{X}, F_{Y}\right)$ and thus recognizes only very severe differences in the level of dependency between summands. In this paper we investigate how distortion risk measures are related to some more elastic measures, namely:

- Pearson's correlation coefficient

$$
r(X, Y)=\frac{\operatorname{Cov}(X, Y)}{\sigma(X) \sigma(Y)}
$$

- Spearman's rank correlation coefficient

$$
\rho(X, Y)=\frac{E\left[F_{X}(X) F_{Y}(Y)\right]-E\left[F_{X}(X)\right] E\left[F_{Y}(Y)\right]}{\sigma\left(F_{X}(X)\right) \sigma\left(F_{Y}(Y)\right)}=r\left(F_{X}(X), F_{Y}(Y)\right)
$$

- Kendall's rank correlation coefficient

$$
\tau(X, Y)=\operatorname{Pr}\left(\left(X-X^{\prime}\right)\left(Y-Y^{\prime}\right)>0\right)-\operatorname{Pr}\left(\left(X-X^{\prime}\right)\left(Y-Y^{\prime}\right)<0\right),
$$

where $(X, Y)$ and $\left(X^{\prime}, Y^{\prime}\right)$ are two independent copies from the considered bivariate distribution.

We show that in general there is no strict relation between distortion risk measures and these measures of dependencies. We prove that for any distortion function $g$ it is possible to find such random pairs with fixed marginals $\left(X_{1}, Y_{1}\right)$ and $\left(X_{2}, Y_{2}\right)$ that

$$
H_{g}\left[X_{1}+Y_{1}\right]>H_{g}\left[X_{2}+Y_{2}\right]
$$

despite

$$
r\left(X_{1}, Y_{1}\right)<r\left(X_{2}, Y_{2}\right) .
$$

Moreover we show that if the selected distortion function is not "too concave" (we formalize this notion in the next section) we can even strengthen the pitfall by showing that also $\rho\left(X_{1}, Y_{1}\right)<\rho\left(X_{2}, Y_{2}\right)$ and $\tau\left(X_{1}, Y_{1}\right)<\tau\left(X_{2}, Y_{2}\right)$.

\section{The main result}

We split our results into two cases: the critical case when $g$ is concave and the easy case of non-concave distortion functions. 


\subsection{Construction for concave distortion functions}

We restrict ourselves only to the case when a distortion function $g:[0,1] \rightarrow[0,1]$ satisfies some additional smoothness conditions. More precisely we will assume the following:

(i) $g(0)=0$ and $g(1)=1$;

(ii) $g$ is piecewise twice continuously differentiable;

(iii) for all $x g^{\prime}(x) \geq 0$ (thus $g$ is nondecreasing) and $g$ is concave;

(iv) $g$ differs from the identity function.

Condition (iv) excludes the trivial case of the expectation. Note that assumption (ii) allows for example piecewise linear distortion functions. In fact in our proof we use only left continuity at 1 and right continuity at 0 of the first derivative.

We start with a helpful technical lemma.

Lemma 1 Let $g$ be an arbitrary function satisfying conditions (i)-(iv). Then there exist real numbers $\alpha_{1}<\alpha_{2}$ in $(0,1)$ and $x \in\left(\frac{1}{2}, 1\right)$ such that $g^{\prime}\left(\alpha_{1}\right)>g^{\prime}\left(\alpha_{2}\right)$ and

$$
(1-x) g^{\prime}\left(\alpha_{1}\right)+x g_{-}^{\prime}(1)>g^{\prime}\left(\alpha_{2}\right) .
$$

If we additionally assume that $-4 g_{-}^{\prime \prime}(1)<g_{+}^{\prime}(0)-g_{-}^{\prime}(1)$ then for (2.1) to hold true we may take $x=\frac{2}{3-\sqrt{\alpha_{2}}}$.

Proof To prove the first part, we start with choosing any $\alpha_{1} \in(0,1)$ such that

$$
g^{\prime}\left(\alpha_{1}\right)>g_{-}^{\prime}(1)>0
$$

(this is always possible in view of conditions (i)-(iv)). For fixed $x \in\left(\frac{1}{2}, 1\right)$ define $\varepsilon=(1-x)\left(g^{\prime}\left(\alpha_{1}\right)-g_{-}^{\prime}(1)\right)>0$. Left continuity of $g^{\prime}$ in 1 implies that it is possible to choose a point $\alpha_{2}$ such that

$$
0<g^{\prime}\left(\alpha_{2}\right)-g_{-}^{\prime}(1)<\varepsilon
$$

Then one gets

$$
g^{\prime}\left(\alpha_{2}\right)<g_{-}^{\prime}(1)+\varepsilon=(1-x) g^{\prime}\left(\alpha_{1}\right)+x g_{-}^{\prime}(1)
$$

Moreover,

$$
g^{\prime}\left(\alpha_{2}\right)-g_{-}^{\prime}(1)<\varepsilon<\frac{g^{\prime}\left(\alpha_{1}\right)-g_{-}^{\prime}(1)}{2}<g^{\prime}\left(\alpha_{1}\right)-g_{-}^{\prime}(1)
$$

and hence

$$
g^{\prime}\left(\alpha_{1}\right)>g^{\prime}\left(\alpha_{2}\right)
$$

which completes the proof of the first part. 
The proof of the second part is a bit more subtle, because here, $x$ is defined as a function of $\alpha_{2}$. Recall that we additionally assume here that

$$
-4 g_{-}^{\prime \prime}(1)<g_{+}^{\prime}(0)-g_{-}^{\prime}(1) .
$$

From continuity of the first derivative it immediately follows that we can choose $\alpha_{1}>0$ that

$$
-4 g_{-}^{\prime \prime}(1)>g^{\prime}\left(\alpha_{1}\right)-g_{-}^{\prime}(1) .
$$

Note that inequality (2.1) which has to be proven can be rewritten as

$$
g^{\prime}\left(\alpha_{1}\right)-g^{\prime}\left(\alpha_{2}\right)>\frac{2}{3-\sqrt{\alpha_{2}}}\left(g^{\prime}\left(\alpha_{1}\right)-g_{-}^{\prime}(1)\right) .
$$

Consider an auxiliary function $f$ defined as follows:

$$
f(p)=g^{\prime}\left(\alpha_{1}\right)-g^{\prime}(p)-\frac{2}{3-\sqrt{p}}\left(g^{\prime}\left(\alpha_{1}\right)-g_{-}^{\prime}(1)\right) .
$$

One can easily check that $f(1)=0$ and

$$
f_{-}^{\prime}(1)=-g_{-}^{\prime \prime}(1)-\frac{1}{4}\left(g^{\prime}\left(\alpha_{1}\right)-g_{-}^{\prime}(1)\right)<0 .
$$

Thus it is possible to choose $\alpha_{2} \in\left(\alpha_{1}, 1\right)$ such that $f\left(\alpha_{2}\right)>0$. Moreover from the identity

$$
g^{\prime}\left(\alpha_{1}\right) \leq g^{\prime}(t) \Rightarrow f(t)<0
$$

we conclude that $g^{\prime}\left(\alpha_{1}\right)>g^{\prime}\left(\alpha_{2}\right)$ what completes the proof of Lemma 1 .

Theorem 2 Let $g$ be an arbitrary function satisfying conditions (i)-(iv). Then there exist univariate discrete distributions $F_{X^{(g)}}, F_{Y^{(g)}}$ and random couples $\left(X_{1}^{(g)}, Y_{1}^{(g)}\right),\left(X_{2}^{(g)}, Y_{2}^{(g)}\right)$ belonging to $R\left(F_{X^{(g)}}, F_{Y^{(g)}}\right)$ such that

(i) $r\left(X_{1}^{(g)}, Y_{1}^{(g)}\right)<r\left(X_{2}^{(g)}, Y_{2}^{(g)}\right)$,

(ii) $H_{g}\left[X_{1}^{(g)}+Y_{1}^{(g)}\right]>H_{g}\left[X_{2}^{(g)}+Y_{2}^{(g)}\right]$.

Moreover under additional assumption that

$$
-4 g_{-}^{\prime \prime}(1)<g_{+}^{\prime}(0)-g_{-}^{\prime}(1)
$$

the random couples can be chosen such that also $\rho\left(X_{1}^{(g)}, Y_{1}^{(g)}\right)<\rho\left(X_{2}^{(g)}, Y_{2}^{(g)}\right)$ and $\tau\left(X_{1}^{(g)}, Y_{1}^{(g)}\right)<\tau\left(X_{2}^{(g)}, Y_{2}^{(g)}\right)$.

Proof Consider two points $0<\alpha_{1}<\alpha_{2}<1$ satisfying the conditions of Lemma 1. Consider the random variables $X^{(g)}$ and $Y^{(g)}$ for which $\operatorname{Pr}\left(X^{(g)}=i\right)=p_{i}$ and $\operatorname{Pr}\left(Y^{(g)}=j\right)=q_{j}$ are given below:

$$
p_{0}=p_{1}=\frac{1-\sqrt{\alpha_{2}}}{2}, \quad p_{2}=\sqrt{\alpha_{2}}
$$


and

$$
q_{0}=1-\frac{\alpha_{1}}{\sqrt{\alpha_{2}}}, \quad q_{1}=\frac{\alpha_{1}}{\sqrt{\alpha_{2}}} .
$$

Furthermore, let $\left(X_{1}^{(g)}, Y_{1}^{(g)}\right)$ be an independent pair with marginal distributions as defined in (2.3) and (2.4), i.e.:

$$
\operatorname{Pr}\left[X_{1}^{(g)}=i, Y_{1}^{(g)}=j\right]=p_{i} q_{j}
$$

The joint distribution of $\left(X_{2}^{(g)}, Y_{2}^{(g)}\right)$ is defined in Table 1 , where $x$ denotes

(i) a fixed number in $\left(\frac{1}{2}, 1\right)$, e.g. $x=\frac{2}{3}$, if $(2.2)$ is not satisfied;

(ii) $x=\frac{2}{3-\sqrt{\alpha_{2}}}$ if $(2.2)$ is satisfied

and $\varepsilon$ is chosen as an arbitrary positive number such that

$$
\varepsilon \leq \min \left(\frac{p_{0} q_{1}}{x}, p_{1} q_{0}, \frac{p_{2} q_{1}}{1-x}\right) .
$$

\begin{tabular}{c|ccc}
\hline & \multicolumn{3}{|c}{$X_{2}^{(g)}$} \\
\hline$Y_{2}^{(g)}$ & 0 & 1 & 2 \\
\hline 0 & $p_{0} q_{0}+x \varepsilon$ & $p_{1} q_{0}-\varepsilon$ & $p_{2} q_{0}+(1-x) \varepsilon$ \\
1 & $p_{0} q_{1}-x \varepsilon$ & $p_{1} q_{1}+\varepsilon$ & $p_{2} q_{1}-(1-x) \varepsilon$ \\
\hline
\end{tabular}

Table 1: The distribution of $\left(X_{2}^{(g)}, Y_{2}^{(g)}\right)$.

Note that in the case when (2.2) is satisfied the following inequalities hold:

$$
1 \geq x>\max \left(\frac{1}{2}, \frac{2 \sqrt{\alpha_{2}}}{1+\sqrt{\alpha_{2}}}, \frac{1+\sqrt{\alpha_{2}}}{3-\sqrt{\alpha_{2}}}\right)
$$

One can immediately verify that $\left(X_{2}^{(g)}, Y_{2}^{(g)}\right) \in R\left(F_{X}, F_{Y}\right)$. Note also that for the first independent pair one has $r\left(X_{1}, Y_{1}\right)=\rho\left(X_{1}, Y_{1}\right)=\tau\left(X_{1}, Y_{1}\right)=0$, which have to be compared to the correlation coefficients of the second pair calculated as follows:

(i) $\operatorname{Cov}\left(X_{2}, Y_{2}\right)=(2 x-1) \varepsilon>0$ and thus also $r\left(X_{2}, Y_{2}\right)>0$;

(ii) From (1.2) we have that

$$
\rho\left(X_{2}, Y_{2}\right)=\frac{\varepsilon\left(1-q_{0}\right)\left((1-x) p_{0}+p_{1}-(1-x)\right)}{\sigma\left(F_{X}(X)\right) \sigma\left(F_{Y}(Y)\right)},
$$

which is positive when $x>\frac{1-p_{0}-p_{1}}{1-p_{0}}$. Combining this with (2.3) we get that $x>\frac{2 \sqrt{\alpha_{2}}}{1+\sqrt{\alpha_{2}}}$ which is in view of (2.6) true in the case when (2.2) holds. 
(iii) A straightforward manipulation on (1.3) leads to the formula:

$$
\begin{aligned}
\tau\left(X_{2}, Y_{2}\right) & =2\left(\left(p_{0} q_{0}+x \varepsilon\right)\left(p_{2} q_{1}-(1-x) \varepsilon\right)+\left(p_{0} q_{0}+x \varepsilon\right)\left(p_{1} q_{1}+\varepsilon\right)\right. \\
& \left.+\left(p_{1} q_{0}-\varepsilon\right)\left(p_{2} q_{1}-(1-x) \varepsilon\right)\right)-2\left(\left(p_{0} q_{1}-x \varepsilon\right)\left(p_{2} q_{0}+(1-x) \varepsilon\right)\right. \\
& \left.+\left(p_{0} q_{1}-x \varepsilon\right)\left(p_{1} q_{0}-\varepsilon\right)+\left(p_{1} q_{1}+\varepsilon\right)\left(p_{2} q_{0}+(1-x) \varepsilon\right)\right) .
\end{aligned}
$$

Note that all expressions without $\varepsilon$ sum up to 0 (this can be also seen from a heuristic argument that for $\varepsilon=0$ one has the independent case when $\tau=0$ ) as well as all expressions with $\varepsilon^{2}$ and thus (after some calculations) the condition for $\tau\left(X_{2}, Y_{2}\right)$ to be positive is equivalent to the inequality

$$
x p_{0}+(2 x-1) p_{1}-(1-x) p_{2}>0,
$$

which - after taking into account $(2.3)$ - gives $x>\frac{1+\sqrt{\alpha_{2}}}{3-\sqrt{\alpha_{2}}}$, which is true in the case when (2.2) holds.

Let us define $S_{1}^{(g)}=X_{1}^{(g)}+X_{1}^{(g)}$ and $S_{2}^{(g)}=X_{2}^{(g)}+Y_{2}^{(g)}$. To complete the proof of Theorem 2, it suffices to prove that

$$
H_{g}\left[S_{1}^{(g)}\right]>H_{g}\left[S_{2}^{(g)}\right] .
$$

We compute the distribution of $S_{1}^{(g)}$ as follows:

$$
\begin{aligned}
f_{1}(2) & =\operatorname{Pr}\left[S_{1}^{(g)}>2\right]=p_{2} q_{1}=\sqrt{\alpha_{2}} \frac{\alpha_{1}}{\sqrt{\alpha_{2}}}=\alpha_{1} \\
f_{1}(1) & =\operatorname{Pr}\left[S_{1}^{(g)}>1\right]=p_{2} q_{1}+p_{1} q_{1}+p_{2} q_{0}=\alpha_{1}+\frac{1-\sqrt{\alpha_{2}}}{2} \\
& =\frac{\alpha_{1}}{\sqrt{\alpha_{2}}}+\sqrt{\alpha_{2}}\left(1-\frac{\alpha_{1}}{\sqrt{\alpha_{2}}}\right)>\sqrt{\alpha_{2}}>\alpha_{2} \\
f_{1}(0) & =\operatorname{Pr}\left[S_{1}^{(g)}>0\right]=1-p_{0} q_{0}<1 .
\end{aligned}
$$

One finds the following expression for the decumulative distribution function:

$$
\bar{F}_{S_{1}^{(g)}}(t)= \begin{cases}1 & \text { for } t<0 \\ f_{1}(k) & \text { for } k \leq t<k+1 \text { and } k=0,1,2 \\ 0 & \text { for } t \geq 3\end{cases}
$$

Now using formula (1.1), we find

$$
H_{g}\left[S_{1}^{(g)}\right]=g\left(f_{1}(0)\right)+g\left(f_{1}(1)\right)+g\left(f_{1}(2)\right) .
$$

Analogously, we define values $f_{2}(k)=\operatorname{Pr}\left[S_{2}^{(g)}>k\right]$ for $k=0,1,2$. We get the following identities:

$$
\begin{aligned}
& f_{2}(2)=f_{1}(2)-(1-x) \varepsilon \\
& f_{2}(1)=f_{1}(1)+\varepsilon \\
& f_{2}(0)=f_{1}(0)-x \varepsilon
\end{aligned}
$$


Thus

$$
H_{g}\left[S_{2}^{(g)}\right]=g\left(f_{1}(0)-x \varepsilon\right)+g\left(f_{1}(1)+\varepsilon\right)+g\left(f_{1}(2)-(1-x) \varepsilon\right) .
$$

After combining (2.11) with (2.12) we see that in order to complete the proof of inequality (2.7) it suffices to prove that

$g\left(f_{1}(2)\right)-g\left(f_{1}(2)-(1-x) \varepsilon\right)+g\left(f_{1}(0)\right)-g\left(f_{1}(0)-x \varepsilon\right)>g\left(f_{1}(1)+\varepsilon\right)-g\left(f_{1}(1)\right)$.

Now let us take a closer insight in differences occurring in inequality (2.13). From the Lagrange Theorem it follows that there exist $0<\varepsilon_{0}, \varepsilon_{1}, \varepsilon_{2}<\varepsilon$ such that the following identities hold:

$$
\begin{gathered}
g\left(f_{1}(0)\right)-g\left(f_{1}(0)-x \varepsilon\right)=x \varepsilon g^{\prime}\left(f_{1}(0)-x \varepsilon_{0}\right)>x g_{-}^{\prime}(1) \varepsilon \\
g\left(f_{1}(1)+\varepsilon\right)-g\left(f_{1}(1)\right)=\varepsilon g^{\prime}\left(f_{1}(1)+\varepsilon_{1}\right)<g^{\prime}\left(\alpha_{2}\right) \varepsilon \\
g\left(f_{1}(2)\right)-g\left(f_{1}(2)-(1-x) \varepsilon\right)=(1-x) \varepsilon g^{\prime}\left(f_{1}(2)-(1-x) \varepsilon_{2}\right)>(1-x) g^{\prime}\left(\alpha_{1}\right) \varepsilon
\end{gathered}
$$

However, from Lemma 1 we find that

$$
(1-x) g^{\prime}\left(\alpha_{1}\right)+x g_{-}^{\prime}(1)>g^{\prime}\left(\alpha_{2}\right)
$$

Multiplying both sides of (2.17) by $\varepsilon$ and combining with inequalities (2.14), (2.15) and (2.16), we get the sequence of inequalities:

$$
\begin{aligned}
g\left(f_{1}(2)\right)-g\left(f_{1}(2)-(1-x)\right. & \varepsilon)+g\left(f_{1}(0)\right)-g\left(f_{1}(0)-x \varepsilon\right) \\
& >(1-x) \varepsilon g^{\prime}\left(\alpha_{1}\right)+x \varepsilon g_{-}^{\prime}(1)>\varepsilon g^{\prime}\left(\alpha_{2}\right) \\
& >g\left(f_{1}(1)+\varepsilon\right)-g\left(f_{1}(1)\right),
\end{aligned}
$$

what completes the proof.

Remark 1 Condition (2.2) requires an additional comment. We believe that this assumption can be somehow released (compare Darkiewicz et al. (2004)), however for our construction this kind of restriction seems to be necessary. Fortunately a lot of distortion functions encountered in practice satisfy this additional limitation. In particular the theorem holds true for all concave piecewise linear functions (e.g. Tail Value-at-Risk admits such representation), because then $g^{\prime \prime}(1)=0$. At the second extreme we have distortion functions for which the first derivative at 0 is infinite and also in this case condition (2.2) follows automatically. The latter case contains other favorite distortion risk measures, like Proportional Hazard Transform (Wang (1995) and Wang (1996)) or its generalization - a Beta distortion risk measure (Wirch and Hardy (2000)). 


\subsection{The non-concave case}

Intuitively, it is clear that the assumption of concavity of $g$ is somehow critical. However in the proof we use this assumption explicitly. In fact, when one releases the assumption of concavity, the construction follows easily from a general theorem proved in Schmeidler (1986).

Theorem 3 Let $B V$ be a set of bounded random variables. Suppose that a functional $H: B V \rightarrow[0, \infty)$

(i) is additive for comonotonic risks;

(ii) preserves the first order stochastic dominance (i.e. $\forall_{t} F_{X}(t) \leq F_{Y}(t) \Rightarrow$ $H[X] \leq H[Y]) ;$

(iii) satisfies $H[1]=1$.

Then there exists a distortion function $h$ such that $H[X]=H_{h}[X]$ for all $X \in B V$. Moreover $H[X+Y] \leq H[X]+H[Y]$ holds for all $X, Y \in B V$ if and only if $h$ is concave.

Proof See e.g. Denneberg (1994), Wang (1996).

Consider a distortion risk measure $H_{g}$ generated by the distortion function $g$ which is not concave. Clearly, $H_{g}$ obeys (i), (ii) and (iii) in the theorem above and therefore we find the following corollary.

Corollary 1 Let $H_{g}$ denote a distortion risk measure generated by a distortion function $g$ which is not concave. Then there exists a bivariate random variable $(X, Y)$ such that $H_{g}[X+Y]>H_{g}[X]+H_{g}[Y]$.

Now it is straightforward to prove the general theorem.

Theorem 4 Let $g$ be an arbitrary non-concave distortion function. Then there exist univariate distributions $F_{X^{(g)}}, F_{Y^{(g)}}$ and bivariate distributions $\left(X_{1}^{(g)}, Y_{1}^{(g)}\right)$, $\left(X_{2}^{(g)}, Y_{2}^{(g)}\right)$ belonging to $R\left(F_{X^{(g)}}, F_{Y^{(g)}}\right)$ such that

(i) $r\left(X_{1}^{(g)}, Y_{1}^{(g)}\right)<r\left(X_{2}^{(g)}, Y_{2}^{(g)}\right)$;

(ii) $\rho\left(X_{1}^{(g)}, Y_{1}^{(g)}\right)<\rho\left(X_{2}^{(g)}, Y_{2}^{(g)}\right)$;

(iii) $\tau\left(X_{1}^{(g)}, Y_{1}^{(g)}\right)<\tau\left(X_{2}^{(g)}, Y_{2}^{(g)}\right)$;

(iv) $H_{g}\left[X_{1}^{(g)}+Y_{1}^{(g)}\right]>H_{g}\left[X_{2}^{(g)}+Y_{2}^{(g)}\right]$. 
Proof If $g$ is not concave, one finds from Corollary 1 that there exists a random couple $(X, Y)$ such that

$$
H_{g}[X+Y]>H_{g}[X]+H_{g}[Y]
$$

On the other hand, for the couple $\left(X^{c}, Y^{c}\right)$ with the same marginal distributions as the couple $(X, Y)$, but with the comonotonic dependency structure, one has that

$$
H_{g}\left[X^{c}+Y^{c}\right]=H_{g}[X]+H_{g}[Y] .
$$

Combining (2.18) with (2.19), one gets

$$
H_{g}[X+Y]>H_{g}\left[X^{c}+Y^{c}\right] .
$$

However we have that $\operatorname{Var}[X+Y]<\operatorname{Var}\left[X^{c}+Y^{c}\right]$ and thus $r(X, Y)<r\left(X^{c}, Y^{c}\right)$ (see Dhaene et al. $(2002 \mathrm{a}, \mathrm{b})$ ). The same is true also for Spearman's $\rho$ and Kendall's $\tau$ because $\rho=\tau=1$ holds true only in the comonotonic case.

Hence, taking $\left(X_{1}^{(g)}, Y_{1}^{(g)}\right)=(X, Y)$ and $\left(X_{2}^{(g)}, Y_{2}^{(g)}\right)=\left(X^{c}, Y^{c}\right)$ leads to the desired result.

\section{References}

[1] Darkiewicz, G.; Dhaene, J.; Goovaerts, M.J. (2004). "Risk measures and dependencies of risks", Brazilian Journal of Probability and Statistics. Submitted.

[2] Denneberg, D. (1994). "Non-additive measure and integral", Kluwer Academic Publishers, Boston.

[3] Dhaene, J.; Denuit, M.; Goovaerts, M.J.; Kaas, R.; Vyncke, D. (2002a). "The concept of comonotonicity in actuarial science and Finance: theory", Insurance: Mathematics \& Economics 31(1), 3-33.

[4] Dhaene, J.; Denuit, M.; Goovaerts, M.J.; Kaas, R.; Vyncke, D. (2002b). "The concept of comonotonicity in actuarial science and finance: applications", Insurance: Mathematics \& Economics, 31(2), 133-161.

[5] Dhaene, J.; Vanduffel, S.; Quihe, T.; Goovaerts, M.J.; Kaas, R.; Vyncke, D. (2004). "Solvency capital, risk measures and comonotonicity: a review", Research Report OR 0416, Department of Applied Economics, K.U.Leuven, pp.33.

[6] Schmeidler, D. (1986). "Integral representation without additivity", Proceedings of the American Mathematical Society 97, 225-261. 
[7] Wang, S. (1995). "Insurance pricing and increased limits ratemaking by proportional hazard transforms", Insurance: Mathematics $\& \mathcal{G}$ Economics 17, 43-54.

[8] Wang, S. (1996). "Premium calculation by transforming the layer premium density", ASTIN Bulletin 26, 71-92.

[9] Wang, S.; Dhaene, J. (1998). "Comonotonicity, correlation order and premium principles", Insurance: Mathematics \& Economics 22, 235-242.

[10] Wang, S. and Young, V.R. (1998). "Ordering risks: expected utility theory versus Yaari's dual theory of risk", Insurance: Mathematics 83 Economics 22, $235-242$.

[11] Wirch, J.L.; Hardy, M.R. (2000). "Ordering of risk measures for capital adequacy", Institute of Insurance and Pension Research, University of Waterloo, Research Report 00-03.

\section{Acknowledgements}

Grzegorz Darkiewicz, Jan Dhaene and Marc Goovaerts acknowledge the financial support of the Onderzoeksfonds K.U.Leuven (GOA/02: Actuariële, financiële en statistische aspecten van afhankelijkheden in verzekerings- en financiële portefeuilles).

The authors would also like to thank Vivien Lewis and Julia Hilgers who helped in translating the summary to German.

\section{Summary}

Distortion Risk Measures for Sums of Random Variables

When we consider random couples $\left(X_{1}, Y_{1}\right)$ and $\left(X_{2}, Y_{2}\right)$, both elements of $R\left(F_{X}, F_{Y}\right)$, relative riskiness of the sums $S_{i}=X_{i}+Y_{i}$ results from dependency structure between the summands. In this paper we investigated the relation between a measure of risk for sums of random variables derived from distortion functions and traditional measures of dependencies like Pearson's $r$, Spearman's $\rho$ and Kendall's $\tau$. In the general case we proved that there is no relation between distortion risk measures and Pearson's $r$. We also showed that for many classes of distortion risk measures (non-concave distortion risk measures, Tail Value-at-Risk, proportional Hazard Transform, beta distortion risk measures and many others) the same holds true additionally for Spearman's $\rho$ and Kendall's $\tau$. These findings aim to illustrate the problem of defining what the right measure of dependency is, and that risk measures widely used in practice are not always consistent with traditional measures of dependencies. 


\section{Zusammenfassung}

Verzerrungsrisikomaße für die Summen von Zufallsvariablen

Beim Betrachten von Paaren von Zufallsvariablen, $\left(X_{1}, Y_{1}\right)$ und $\left(X_{2}, Y_{2}\right)$, beide Elemente von $R\left(F_{X}, F_{Y}\right)$, wird deutlich, dass das relative Risiko der Summen $S_{i}=X_{i}+Y_{i}$ sich aus der Abhängigkeitsstruktur zwischen den Summanden ergibt. In dieser Studie untersuchen wir die Beziehung zwischen einem aus Verzerrungsfunktionen hergeleiteten Risikomaß für die Summen von Zufallsvariablen auf der einen Seite, und traditionellen Abhängigkeitsmaßen wie Pearsons $r$, Spearmans $\rho$ und Kendalls $\tau$ auf der anderen Seite. Wir zeigen auf, dass im Allgemeinfall kein Zusammenhang besteht zwischen dem Verzerrungsrisikomaß und Pearsons $r$. Desweiteren zeigen wir, dass für viele Gruppen von Verzerrungsrisikomaßen (nicht-konkave Verzerrungsrisikomaße, Tail Value-at-Risk, proportionaler Hazard Transform, Beta Verzerrungsrisikomaße und viele andere), außerdem das gleiche für Spearmans $\rho$ and Kendalls $\tau$ gilt. Diese Ergebnisse veranschaulichen zum einen, dass das richtige Abhängigkeitsmaß schwer definierbar ist und zum anderen, dass die weit verbreiteten Risikomaße nicht immer übereinstimmen mit traditionellen Abhängigkeitsmaßen. 
